\title{
CONVECTIVE OVERSHOOT AND UPPER BOUNDARY CONDITIONS
}

\author{
A. Nesis \\ Kiepenheuer-Institut für Sonnenphysik \\ Schöneckstr. 6 \\ West-Germany
}

\begin{abstract}
Calculations of the frequency of solar oscillation are sensitive to the upper boundary conditions of the model. Our investigations of the velocity fields of the overshoot layers (photosphere) have shown that there is a minimum of velocity at about $200 \mathrm{~km}$ above $\tau=1$ : We suggest that this minimum provides a natural upper boundary condition for the calculations of solar oscillation. The propagation of sound in these layers has to be regarded as a propagation in a turbulent medium.
\end{abstract}

Calculations of solar oscillations are sensitive to the upper boundary conditions as pointed out by Ulrich and Rhodes (1983) and Stix (1986). Especially the transition from $\tau=1$ to $\tau=10^{-4}$ modifies the frequency of the solar oscillations in calculations substantially. How can this modification be understood? The layers between the levels $\tau=1$ and $\tau=10^{-4}$ represent the photospheric layers between the deepest observable layers and those of the temperature minimum, and are well investigated. From the dynamical point of view, the layers directly above $\tau=1$ are regarded as the overshoot layers of solar granulation, i.e. as the upper layers of the convective zone. Thus, the physical properties of these layers have to be taken into consideration, because they could modify in a sensitive manner the computations of solar oscillation.

The dynamics of these layers determine also the velocity of sound and its propagation in these layers: the existence of a turbulence field in these layers makes the determination of velocity of sound difficult. In the inversion problem the photospheric layers are not taken into consideration (asymptotic case). However, the gradient of the real sound velocity with the depth in the overshoot layers affects the calculations using the inversion technique by Gough (1984). Fig. 1 shows the variation of a hypothetical model of $c^{2}$ and its $1 / c$ as a function of $r / R$ ( $R$ : is the solar radius). The values of $1 / \mathrm{c}$ increase deeply in the range of the overshoot layers in the photosphere, and thus, demonstrate the importance of these layers for boundary conditions, and for the determination of sound velocity and propagation.

The dynamics of the overshoot layers, i.e. their vertical and horizontal fields, were investigated by means of spectrograms taken with the 


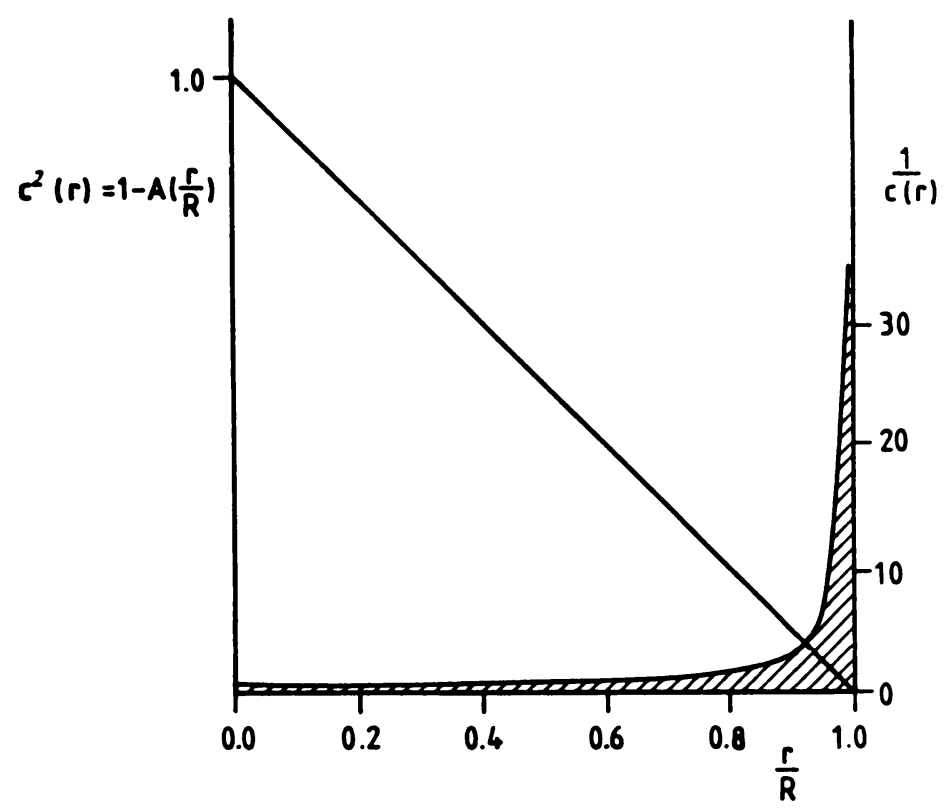

Figure 1: Hypothetical linear variation of velocity of sound $\left(c^{2}: c^{2}=\right.$ $1+A(r / R)$ ) as a function of $r / R$ ( $r$ : distance from the solar center, $R$ : solar radius). The variation of $1 / c$ with $r / R$ demonstrates the importance of the outer solar layers (photosphere) for the calculations of the frequency of solar oscillation: because of the steep increase of 1/c towards the solar surface $(r / R \rightarrow 1)$, the integral $(1 / c) d r$ is strongly weighted by the outer solar layers.

balloon-borne spectrostratoscop. The variation of the vertical and horizontal velocities with the height in the atmosphere were determined by analyzing absorption lines of different strength. The horizontal velocity was obtained from spectrograms taken near the solar limb. By comparing the velocity fields at the higher layers with those of $\tau=1$, using coherence analysis, it was possible to determine, up to which the convective (granular) overshoot can be observed (Nesis, Durrant, Mattig, 1984). It was also found that the horizontal and vertical velocity fields are independent, and that they can not be regarded as components of the convective motion. The variation of the vertical and the horizontal velocity fields with the height are about the same. As Fig. 2 (left) shows, the velocity decreases up to a height of about $200 \mathrm{~km}$ above $\tau=1$ and increases again towards higher levels. The schematic presentation in Fig. 2 (right) demonstrates that the layers between $\boldsymbol{C}=1$ and the minima are considered as overshoot (boundary) layers (Nesis, 1984). The decrease of the horizontal velocity with the height implies the existence 
of shear stress, which produces vorticity and turbulence. Therefore, we have to assume that these overshoot layers cannot be considered as uniform and isentropic. The decrease of the vertical velocity in these layers, together with the results of the coherence analysis indicate that the ordered granular (convective) motions run out in the overshoot (boundary) layers and induce here secondary motions such as turbulence and gravity- or pressure-waves.

We suggest that the layers at which the minima of the velocity fields are observed, can be regarded as the natural upper boundary for the calculation of solar oscillation. It can be assumed that these layers which lie between $\tau=1$ and $\mathrm{ca}$. $200 \mathrm{~km}$ above $\tau=1$ are in local thermodynamic equilibrium (LTE). On the other hand, the model calculations for solar oscillation require that the disturbances of, for example, pressure $\Delta p$ and density $\Delta \rho$ have to become equal to zero at the upper boundary of the model. This could be assumed for the layers in which the velocity minima are found. Furthermore, we expect, that a detailed knowledge about the physics of the overshoot layers can help us to study the behaviour of the sound propagation in the turbulent media of the overshoot (boundary) layers, and to calculate the gradient of sound velocity with the depth in the deep photosphere. This requires the knowledge of the variation of pressure fluctuations $\Delta p$ with the height in

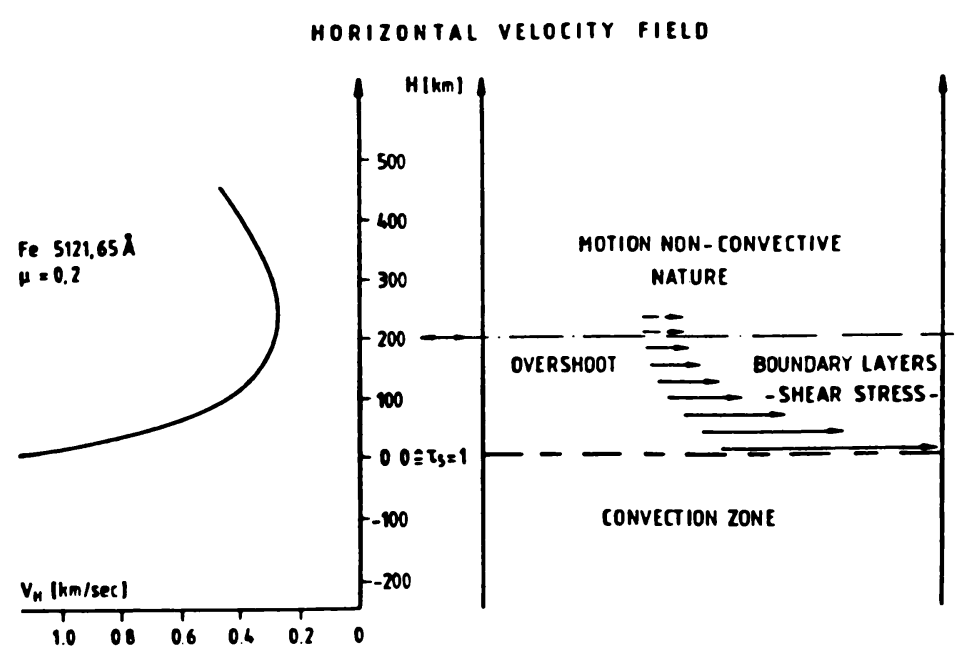

Figure 2: Variations of the horizontal small scale rms-velocity with the height in the deep photosphere (Nesis, 1984). The minimum of velocity is considered to be the upper limit of the overshoot layers. They build up a boundary layer in which horizontal shear stress is found. We suggest that the minimum can be regarded as a natural boundary condition for calculations of solar oscillations. The propagation of sound in these layers has to be considered as a propagation in a turbulent medium. 
the deep photosphere. Considerations about horizontal fluctuation of pressure in these layers can be found also in Kneer (1985). Observations which could give us this information are performed.

\section{REFERENCES}

Gough, D.O.: 1984, Phil. Trans. R. Soc. Lond. A313, 27

Kneer, F.: 1984, in Small-Scale Dynamical Processes in Quiet Atmospheres ed. S.L. Keil, (National Solar Observatory), p. 110

Nesis, A., Durrant, C.J., Mattig, W.: 1984, in Small-Scale Dynamical Processes in Quiet Atmospheres, ed. S.L. Keil, (National Solar Observatory), p. 243

Nesis, A.: 1984, The Hydromagnetics of the Sun, ESA SP-220, p. 203

Stix, M.: 1986, private communication

Ulrich, R.K., Rhodes, E.J.: 1983, Astrophys. J. 265, 551 